\section{SATISFACTION WITH THE HEALTH CARE SYSTEM IN FAMILIES WITH CHILDREN WITH MENTAL OR PHYSICAL DISABILITIES AND CHRONIC DISEASE}

\author{
A. Graungaard ${ }^{1}$, S. Dührkop Hjort ${ }^{1}$, L. Skov², \\ J.S. Andersen ${ }^{1}$ \\ ${ }^{1}$ Department of General Practice, University \\ of Copenhagen, Copenhagen, ${ }^{2}$ Department of \\ Paediatrics, Glostrup University Hospital, \\ Glostrup, Denmark
}

Background and aims: Families with children with chronic conditions face a broad range of health care professionals and facilities in the care-taking of their child. Parents' satisfaction is known to be related to the family-centeredness of the service organization and to the personal relation to the health professionals. Parental satisfaction with health services is often found to be low. This study aims at examining the contacts the child had to the health care system, parental satisfaction with services and if satisfaction was related to the child's diagnosis.

Methods: A cross-section questionnaire study was conducted in the out-patient settings of a large university hospital. Three diagnostic groups with different symptoms and level of needed care were chosen as; diabetes, Tourettes syndrome and cerebral palsy, and 40 parents in each group completed a questionnaire. Parents were indentified through their up-coming appointments with the pediatric department. A pilot study of 10 parents was conducted. The questionnaire contained the MPOC-20 questionnaire translated and validated into Danish, and a range of other questions related to the local organization of health care for pediatric patients including number of contacts, availability of medical care in acute conditions, provision of different kinds of therapy, vaccinations and contact with the family doctor.

Results: Data are currently analyzed and results will be available in a few months. The pilot study showed that parents were more dissatisfied with the service at the time of diagnosis than later and that involvement of the general practitioner was very infrequent.

\section{WORK PROFILE OF BELGIAN PAEDIATRICIANS IN REGIONAL AND UNIVERSITY HOSPITALS}

P.L. Alliet ${ }^{1,2}$, G. Verellen², M. Alexander ${ }^{2}$, D. Deliège ${ }^{3}$, C. Artoisenet ${ }^{3}$

${ }^{1}$ Paediatrics, Jessa Hospital, Hasselt, ${ }^{2}$ Belgian Academy of Paediatrics, ${ }^{3}$ School of Public Health, University of Louvain, Brussels, Belgium

Introduction: As in other European countries, Belgian hospitals encounter difficulties in recruiting paediatricians. In order to better understand the underlying problems and the needs, it is mandatory to have an insight in the work profile of paediatricians.

Methods: In 2007, a questionnaire was sent to the head of the department of paediatrics in all Belgian hospitals. Their staff members were asked to fill in a detailed time sheet during one week in the period March 19-May 1st. Data were encoded by two separate persons and compared through a software program.

Results: The response rate was $66 \%$. The mean total professional time (weekdays 7-21 h) was 50 h 16 min (male 52 h 51 min and female 48 h 20 min). There is no difference according to the (non) university status. The mean total professional time on saturday is 6 hours. Paediatricians working in a regional hospital spent more time in clinical activities outside the hospital (11 h 24 min vs. 2 h 19 min) and less in management and research/teaching activities ( 6 h 12 min and 0 h 52 min vs 12 h 29 min and $5 \mathrm{~h} 10 \mathrm{~min}$ ).

Conclusion: The mean total professional time during the weekdays is $50 \mathrm{~h} 16 \mathrm{~min}$. The mean professional time on Saturday is 6 hours. Paediatricians working in a regional department spend more time in clinical activities outside the hospital and less in management and research/teaching activities. Female paediatricians are working half a day less than their male colleagues. 